visitors, may have only tenuous links with them Other attached professionals, such as social workers, mental health nurses, and clinical psychologists, also have only sporadic contact in most cases.

The problem is not only semantic. The phrase "primary care" creates an illusion of managed, coherent, multidisciplinary institutions analogous to those in secondary care. This illusion allows the government, managers, and hospital consultants to believe that primary care (namely, general practice) is ready to meet the vast demands of decentralisation of the NHS as outpatient clinics shrink and admissions to hospital become shorter.

In reality, there are simply two clinical professions that work in day to day collaboration in most general practices. The people in these professions are doctors and practice nurses. The only other members of the general practice team are practice managers, secretaries, receptionists, caretakers, and cleaners. General practice is, in effect, a cottage industry. The idea that you can decant one of the world's largest industrial organisationsNHS hospital care-into something called primary care is largely a delusion.

The truth is that most of the primary care sector does not do general practice and that genera practice cannot remotely meet the demands being asked of primary care. I suggest that when we mean general practice we should say general practice. Like Pearson and Jones, we should be realistic about what it is and is not.

JM N LAUNER Forest Road Group Practice, General practitioner

London N9 8RZ

1 Pearson $\mathrm{P}$, Jones $\mathrm{K}$. The primary health care non-team? $B M$ 1994;309:1387-8. (26 November.)

2 Evans KL. Diagnosis and management of sinusitis. BMF 1994 309:1415-22.

\section{Electronic health records}

EDrToR,-I fear that Dipak Kalra seems to have missed what is going on in computing in general practice in Britain while concentrating on other European countries.

Kalra says that "EDIFACT will soon be used in primary care to communicate fee claims." In fact, links between general practices and family health services authorities for registration have functioned for at least two years, and last September my practice completed the first full year of using the system for item of service claims and reimbursement.

Only three software systems for general practice passed initial conformance testing for item of service links. Our practice runs ESUG (Exeter Software Users Group) and in the first year of linkage had 3856 item of service claims. This system should save our family health services authority in Cleveland an extraordinary amount of time. The authority estimates that it takes an average of four minutes to process a paper claim. We should have saved the authority about 280 person hours in one year. Across the country the savings would be enormous.

Kalra identifies the need for an agreed patient database for this kind of project to work. There were some 16000 mismatches of data between our records and those of the family health services authority. These were mostly incorrect spaces or mismatches of punctuation. While we wait for European agreements local agreements may be reached, and computer programs can now generate agreed databases.

The full benefits to general practice of electronic linkage will not be realised until the Department of Health agrees to abandon paper claim forms and signatures and accepts that computer claims (with proper audit trails and good software) are less open to fraud than claims on paper. The main beneficiary of links so far is the family health services authority. If it does not fund links fully it will lose that benefit.

It seems extraordinary that huge European projects with vast funding are taking place when the project linking general practices with family health services authorities, which is working well, will result in potentially large financial savings, and has major potential for leading to improvements in demographic data and planning, is not being backed to the hilt.

Norton Medical Centre,

J R THORNHAM

Norton,

Stockton on Tees TS20 1AN

1 Kalra D. Electronic health records: the European scene. $B M \mathcal{F}$ 1994;309:1358-61. (19 November.)

\section{Problems of overseas adoption}

EDrToR,-Rupert Robin proposes that general practitioners should advise infertile couples that adoption is a suitable "treatment." $\mathrm{He}$ also suggests that adoption in such cases will save NHS resources and at the same time help the deserving orphans of Third World countries. If only life were this simple and solutions to complex problems so easy to find. Adoption is not simple, therapeutic, or cost effective. It is a totally child centred process in which decisions are made only in the best interests of a particular child. When being assessed as potential adopters, couples have no automatic rights to adopt and all that matters is their ability to parent and meet the needs of a very disadvantaged child. When this process is properly done it is far from cost effective. The average costs of a full assessment vary between $£ 6000$ and $£ 10000$.

Adoption is not for everyone. Most adopted children in this country come from backgrounds of abuse and neglect, need to be placed in sibling groups, or are of mixed race. They require very special adopters to meet their needs and heal their emotional damage. It is to avoid these difficult children that couples often resort to adopting babies from other countries. These babies often have birth families who are alive and who in better circumstances would be capable of looking after them. The babies may have undiagnosed medical and developmental needs. They will grow up to be teenagers who will almost certainly ask hard questions about their birth families, their origins and culture, and why they have to travel thousands of miles to find the answers. I wonder how they will view this simple solution. MARY MATHER
Consultant community paediatrician Greenwich Healthcare Trust, London SE18 3RS

1 Robin R. Overseas adoption. BMf 1994;309:1516. (3 December.)

\section{Allergy to colophony}

EDrror,-A D Burden and W N Morley report a case of allergy to colophony. We recently treated a 9 year old boy who presented with inflammation around a periungual wart on the left middle finger after two days' treatment with salicylic acid (Cuplex). The treatment was stopped and the finger dressed with a tubular bandage taped to the hand. Two days later the child was admitted to a surgical ward with a swollen left hand and blistering of the skin that was in contact with adhesive tape; cellulitis was diagnosed (figure). Within hours of his admission itchy blisters developed under adhesive tape around an intravenous cannula. Subsequent patch testing confirmed contact allergy to colophony, a constituent of both the wart treatment

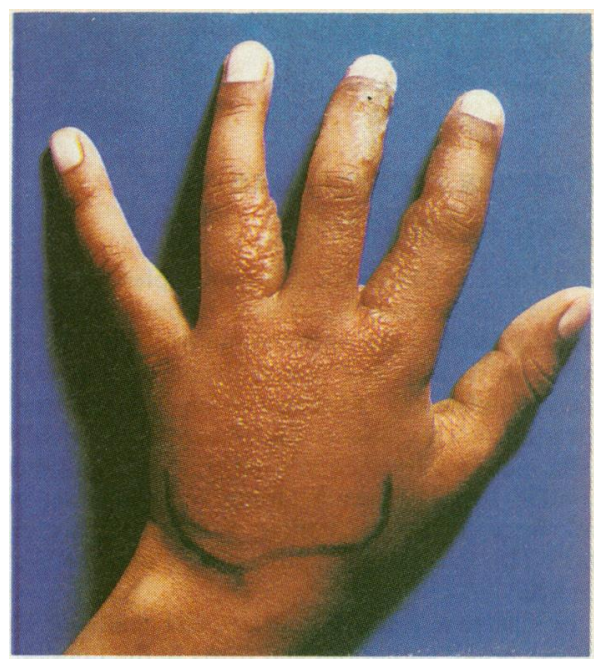

Allergic reaction to colophony

and the adhesive tape. The swelling and blisters resolved rapidly with treatment with a potent topical corticosteroid.

CELIA MOSS

Consultant dermatologis

KATHLEEN BERRY

Children's Hospital, Consultant in accident and emergency

Birmingham B16 8ET

1 Burden AD, Morley WN. Minerva. BMF 1995;310:202 (21 January.)

\section{Leeches}

EDrTor,-Anders Baerheim and Hogne Sandvik observed the effect of ale, garlic, and soured cream on the appetite of leeches. ${ }^{1}$ The three leeches that I met in Tasmania recently were not adversely affected by gorging on a paronychia of the thumb filled with pus. During a hiking holiday through the Cradle Mountain area of Tasmania leeches were applied to my colleague's paronychia. They gave instant relief from pain and reduced the swelling dramatically. The leeches' production of local anaesthesia was such that within seconds the throbbing had disappeared and minutes later the thumb had returned to normal size, so that surgery was unnecessary. The thread-like leeches became the size, shape, and colour of blue grapes. They were detached from the thumb by use of saline solution and were returned to the wilderness.

C EDMUND GRAHAM

Bondi Junction 2022,

Orthopaedic surgeon

Australia

1 Baerheim A, Sandvik H. Effect of ale, garlic, and soured cream on the appetite of leeches. BMf 1994;309:1689. (24-31 December.)

\section{Corrections}

\section{Biological washing powders as allergens}

A typesetting error occurred in this letter by Michael Flindt (21 January, p 195). The second half of the firs sentence of the final paragraph should have read: "it is particularly unfortunate when patients are symptomatically treated for asthma while they continue unknowingly [not knowingly, as printed] to be exposed to a specific allergen."

Interpretation of electrocardiograms by doctors Owing to an editorial error the authors' address was omitted from this letter by P A Woodmansey and colleagues (18 February, p 468). Their address is the department of cardiology, Royal Hallamshire Hospital, Sheffield S10 2JF. 